\title{
ASSESSMENT OF HYDROCEPHALUS IN CHILDREN BASED ON DIGITAL IMAGE PROCESSING AND ANALYSIS
}

\author{
ANNA FABIJAŃSKA *, TOMASZ WĘGLIŃSKI* , KRZYSZTOF ZAKRZEWSKI **, \\ EMILIA NOWOSŁAWSKA** \\ * Institute of Applied Computer Science \\ Łódź University of Technology, Stefanowskiego 18/22, 90-924 Łódź, Poland \\ e-mail: \{an_fab, tweglinski\}@kis.p.lodz.pl \\ ** Department of Neurosurgery \\ Polish Mother’s Memorial Hospital, Research Institute in Łódź, Rzgowska 281/289, 93-338 Łódź, Poland
}

\begin{abstract}
Hydrocephalus is a pathological condition of the central nervous system which often affects neonates and young children. It manifests itself as an abnormal accumulation of cerebrospinal fluid within the ventricular system of the brain with its subsequent progression. One of the most important diagnostic methods of identifying hydrocephalus is Computer Tomography (CT). The enlarged ventricular system is clearly visible on CT scans. However, the assessment of the disease progress usually relies on the radiologist's judgment and manual measurements, which are subjective, cumbersome and have limited accuracy. Therefore, this paper regards the problem of semi-automatic assessment of hydrocephalus using image processing and analysis algorithms. In particular, automated determination of popular indices of the disease progress is considered. Algorithms for the detection, semi-automatic segmentation and numerical description of the lesion are proposed. Specifically, the disease progress is determined using shape analysis algorithms. Numerical results provided by the introduced methods are presented and compared with those calculated manually by a radiologist and a trained operator. The comparison proves the correctness of the introduced approach.
\end{abstract}

Keywords: hydrocephalus, computed tomography, image segmentation, Evans index, frontal and occipital horn ratio, ventricular angle, frontal horn radius.

\section{Introduction}

Hydrocephalus is a common pathological condition of the central nervous system, resulting from disturbances in CerebroSpinal Fluid (CSF) circulation. It involves excessive accumulation of the CSF within the ventricular system of the brain and causes it to expand. The extent of ventricles expansion is often significant and requires neurosurgery.

In the assessment of hydrocephalus the dominant role is played by radiological techniques, including Computer Tomography (CT) and Magnetic Resonance Imaging (MRI). These examinations produce three dimensional (3D), volumetric images of the brain. However, the assessment of hydrocephalus is still mainly manual. It is commonly based on qualitative analysis of the lesion size, combined with additional measurements of its characteristic dimensions. These are performed manually, on the most representative CT or MRI slices, and are encum- bered with 'human errors' resulting from personal skills and experience. Since both the selection of the most representative slice and the calculation of the characteristic dimensions are performed by different specialists, such assessment is very subjective. As a result, the determination of the corresponding slices and characteristic dimensions in two investigations become difficult. Consequently, the repeatability of the assessment may be limited.

Having in mind the above mentioned limitations of the existing methods, this paper proposes image processing and analysis algorithms dedicated to the assessment of hydrocephalus based on CT scans. The introduced algorithms facilitate and automate the evaluation of the disease progress, making it more objective and less humandependent. In particular, the proposed approach firstly performs semiautomatic segmentation of the CSF and the intracranial brain area. Next, based on the most representative CT slice, the popular indices of the disease progress 
are automatically computed.

The paper is organized as follows. Firstly, Section 2 provides basic information about CT based diagnosis of hydrocephalus. This is followed in Section 3 by a review of the related works concerning application of image processing and analysis for segmentation of the cerebrospinal fluid and assessment of hydrocephalus. Section 4 describes the introduced algorithms and methods. Results provided by the proposed approach are presented and discussed in Section 5. Finally, Section 6 concludes the paper.

\section{Medical background}

The Central Nervous System (CNS) in humans consists of the brain and the spinal cord. The brain contains a ventricular system. It consists of two large lateral ventricles (one in either hemisphere) and two smaller ones (the third and the fourth one). The ventricular system contains the cerebrospinal fluid which baths the surface of the CNS and is permanently circulating. In hydrocephalus, a dynamic balance of the CSF is excessively accumulated within the ventricular system. As a result, the ventricles expand and compress the surrounding brain tissue, producing harmful intracranial hypertension. A normal and an enlarged ventricular system of a brain are sketched in Figs. 1(a) and 1 (b), respectively.

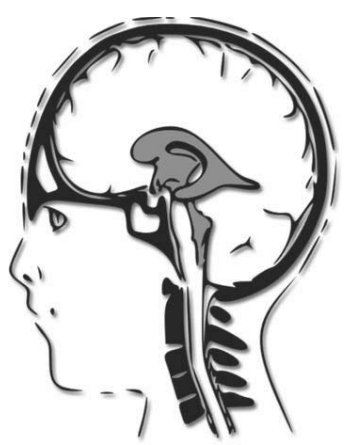

(a)

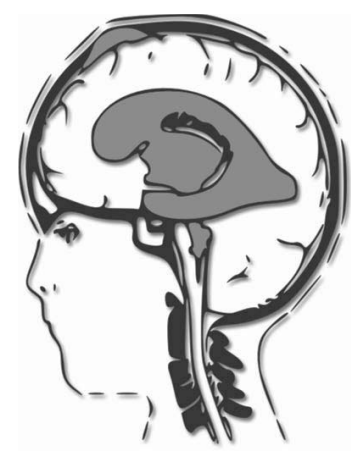

(b)
Fig. 1. Normal (a) and enlarged (b) ventricular system.

Pathological changes due to hydrocephalus can be easily observed in CT scans. In particular, the enlarged ventricular system filled with the CSF has significantly lower intensity than the adjacent brain tissue. This is shown in Fig. 2, which compares CT slices from the scan of a normal brain (Fig. 2 (a)) and a brain affected by hydrocephalus (Fig. 2(b)). The edge of the brain tissue is outlined by the dark gray line. The edges of the brain region affected by hydrocephalus are highlighted by the light gray line.

The diagnosis of hydrocephalus is usually based on visual assessment. However, in some cases, the additional planar measurements are performed. The ma-

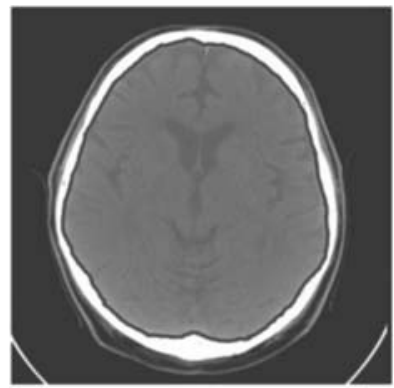

(a)

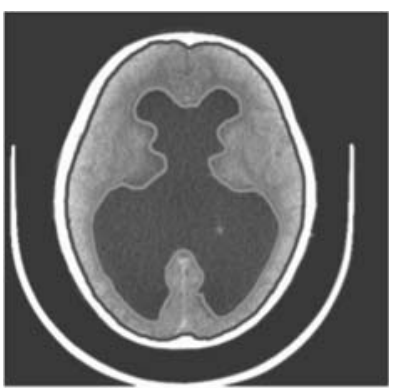

(b)
Fig. 2. Sample CT cross sections of the brain: healthy brain (a), brain affected by hydrocephalus (b).

jor parameters used to classify hydrocephalus include (Barkovich, 2005) the Evans ratio, the frontal and occipital horn ratio, the ventricular angle and the frontal horn radius.

The Evans Ratio (ER) (Synek et al., 1976; Lie et al., 2002) is the ratio of the maximal width of the frontal horns to the maximal width of the inner skull. Using symbols shown in Fig. 3 a), it can be defined by

$$
\mathrm{ER}=\frac{d_{f h}}{\phi_{b_{\max }}} .
$$

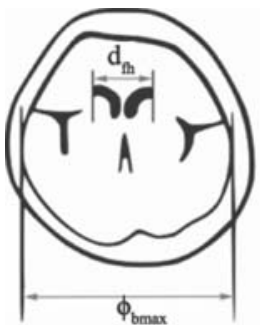

(a)

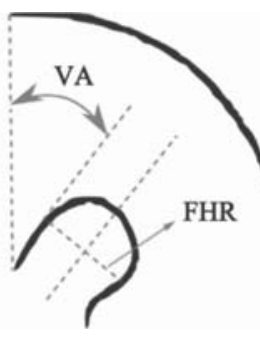

(b)

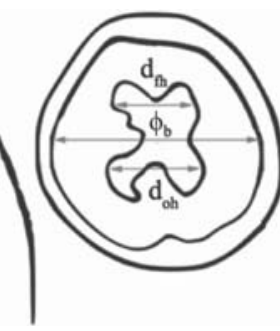

(c)
Fig. 3. Characteristic dimensions of the brain ventricular system used for the assessment of hydrocephalus.

The Frontal and Occipital Horn Ratio (FOHR) (O'Hayon et al., 1998) is the ratio of the sum of the maximal width of the anterior and the occipital horns to the width of the inner skull at the level of the minimal width of the ventricular system. Using symbols shown in Fig. 3. (c), it can be defined by

$$
\mathrm{FOHR}=\frac{d_{f h}+d_{o h}}{2 \phi_{b}} .
$$

The Frontal Horn Radius (FHR) is determined by measuring the widest diameter of the frontal horns taken at the angle of $90^{\circ}$ to the long axis of the frontal horn (see Fig. 3(b)). As hydrocephalus causes concentric enlargement of the frontal horns with their rounded configuration, 
it also causes an increase in the FHR. In turn, an increase in the FHR causes the narrowing of the Ventricular Angle (VA), i.e., the angle made by the anterior or superior margins of the frontal horn at the level of the foramina of Monro (see Fig. 3 (c)) (adapted from the works of Hamano et al. (1993) and Lie et al. (2002)).

All the above mentioned parameters used for the assessment of hydrocephalus are determined based on the most representative slice. Due to the complexity of brain structures, this slice changes depending on the particular measure. Therefore, in the proposed approach, the most representative slice must be selected manually by the radiologist.

\section{Related works}

There are numerous approaches dedicated to the segmentation of the CSF and the brain ventricular system from CT and MRI scans. However, only few works dedicated to application of image processing and analysis in the quantitative assessment of hydrocephalus have been reported so far. These works are generally new and there is no complete research in this area.

So far, much attention of the research in the assessment of hydrocephalus has been paid to the development of algorithms for segmentation of the cerebrospinal fluid. This is a very challenging task due to the complexity and large variations in brain structures. Therefore, in most cases, the existing approaches are based on manual or semi-manual extraction of the CSF or automatic segmentation using basic image processing methods. Among segmentation methods of brain lesion caused by hydrocephalus, the dominant are intensity-based approaches. In particular, Gupta et al. (2010) proposed adaptive image thresholding based on heuristics on the shape of a CT histogram and guided by the connectivity and the domain knowledge. A similar approach was also proposed by Ruttimann et al. (1993), who applied a local thresholding technique for CSF segmentation based on maximumentropy principles. The obtained results were also compared with those of semi-manual segmentation performed by the operator.

Intensity-based clustering (Kulczycki and Charytanowicz, 2010; Frąckiewicz and Palus, 2011) was applied by Halberstadt and Douglas (2005). There are also some approaches which apply threshold based region growing for segmentation of the CSF (Kanayama et al., 1998). In the approach proposed by Schnack et al. (2001) the growth of a region is additionally supported by mathematical morphology. More advanced methods applied for segmentation of the cerebrospinal fluid include watersheds (Luo et al., 2010), active contours (Zang et al., 2010) and level sets (Bosnjak et al., 2007; Butman and Linguraru, 2008). The latter is, however, prohibitively time consuming for practical use.
There are also some reports about semi-manual segmentation of the CSF using ImageJ (Hiraoka et al., 2010) (i.e., free software for image processing) or manual threshold setting in the Qbrain software (Ambarki et al., 2011). However, in all the above mentioned cases, image segmentation is performed separately (slice-by-slice) and volumetric information contained within the brain scan is ignored.

There are also some approaches which use results of image segmentation to estimate the volume of the cerebrospinal fluid and its relation to the brain volume (e.g., Halberstadt and Douglas, 2005; Butman and Linguraru, 2008; Luo et al., 2010; Pustkova et al., 2010) or our previous approach (Węgliński and Fabijańska, 2012a). In all these approaches the volumes are simply determined by counting the number of pixels included into the binary images after segmentation and then multiplying it by a volume of a single pixel. To the best of our knowledge, among the reported approaches, only a few regard the assessment of hydrocephalus using the measures mentioned in Section 2. Some ideas for automatic assessment of the lesion using these indices were proposed by O'Hayon et al. (1998) and Lie et al. (2002). However, the authors provided few details about the employed image processing and analysis algorithms. Having in mind the poorness and limitations of the existing approaches for quantitative assessment of hydrocephalus, in this paper a set of image processing and analysis algorithms dedicated to the problem considered is proposed. In particular, algorithms for lesion segmentation and automatic determination of the popular indices of the disease progress are introduced.

\section{Proposed approach}

The proposed approach consists of two main stages, namely, image processing and image analysis. The former includes image enhancement (preprocessing) and image segmentation. Image segmentation aims at segmentation of the intracranial brain area and of the CSF. In the latter the indices of the hydrocephalus progress are determined based on binary images after segmentation. Selected parameters are computed for the most representative slice indicated manually.

Details of methods used for image segmentation and image analysis are given in the following subsections.

\subsection{Image processing.}

4.1.1. Image preprocessing. During the preprocessing, input data are firstly transformed into Hounsfield Units (HUs). The transformation is performed by applying Rescale Slope $\left(R_{S}\right)$ and Rescale Intercept $\left(R_{I}\right)$ to each pixel in accordance with the following equation:

$$
I_{2}=I_{1} R_{S}+R_{I}[\mathrm{HU}]
$$


where $I_{1}$ is the input pixel intensity, $I_{2}$ is the output pixel intensity given in Hounsfield units, and Rescale Slope and Rescale Intercept are parameters stored in a DICOM header (DICOM, 2004).

Next, pixel intensities are normalized to 12-bit unsigned integers (0-4095) in accordance with

$$
I_{3}= \begin{cases}0 & \text { for } I_{2} \leq h \\ 4095 \frac{I_{2}-z+0,5}{w-0,5} & \text { for } h<I_{2}<g \\ 4095 & \text { for } I_{2} \geq g\end{cases}
$$

where

$$
\begin{gathered}
h=z-0,5(2-w), \\
g=z-0,5 w
\end{gathered}
$$

and $w$ and $z$ stand for the window width and the window center, respectively, and are given in a DICOM header (DICOM, 2004).

The result of preprocessing the brain slice from Fig. 4(a) is shown in Fig. 4(b). It can be easily seen that preprocessing highlights information connected with brain lesion due to hydrocephalus. This significantly facilitates further image processing and analysis.

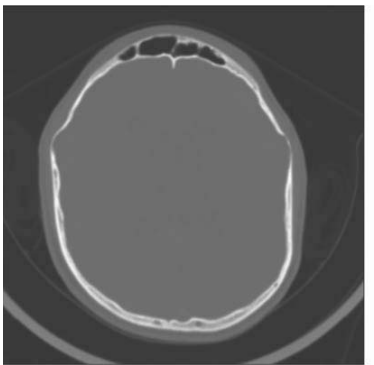

(a)

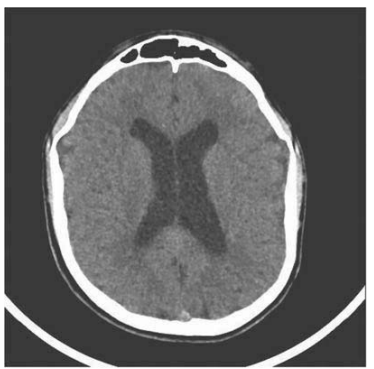

(b)
Fig. 4. Results of image preprocessing: original image (a), enhanced image (b).

4.1.2. Segmentation of the intracranial brain. Ordinary CT scans contain image data of the whole brain from the top of the skull down to the beginning of the spinal cord. Therefore, some of the slices from output datasets usually contain additional information corresponding to skin, mouth, eyes or nose. Since these structures are characterized by similar intensity levels as the brain or the CSF, they often cause some failures in automatic segmentation of the intracranial brain area. To solve this problem, multilevel thresholding based on the estimated binary masks is proposed to extract the intracranial brain.

The presented algorithm is fully automatic and works in two main steps. Firstly, in order to remove low intensity pixels connected with the background and high intensity pixels connected with skull, the following simple thresholding is performed:

$$
I_{B}= \begin{cases}1 & \text { for } I_{3} \in\left[T_{1}, T_{2}\right], \\ 0 & \text { for } I_{3} \notin\left[0, T_{1}\right) \cup\left(T_{2}, 4095\right],\end{cases}
$$

where $T_{1}$ and $T_{2}$ are thresholds determined based on the intensity distribution within the input CT dataset. After intensity normalization (see Section 4.1.1), $T_{1}$ corresponds to the permitted intensities for "air" in the range [0-24). $T_{2}$ corresponds to the highest intensity found within the image histogram and belongs to "dense bone".

Next, pixels associated with the skull (i.e., where $I_{3} \geq T_{2}$ ) are used as a boundary for a binary mask covering the whole intracranial brain area. In order to obtain a properly closed and filled mask, a combination of morphological closing and hole-filling is used. Finally, the intracranial brain region is obtained by multiplying the input image by the binary mask. Results of threshold-based segmentation of the intracranial brain area are shown in Fig. 5 In particular, Fig. 5(a) shows the input image, Fig. 5(b) presents the binary mask, and Fig. 5(c) shows the segmented intracranial brain region.

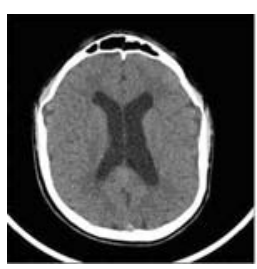

(a)

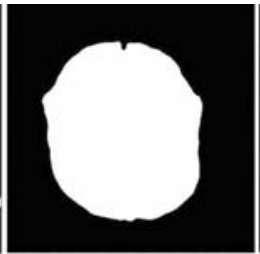

(b)

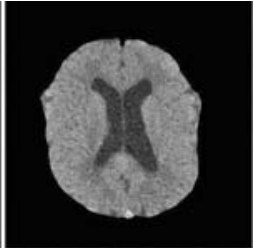

(c)
Fig. 5. Result of segmentation of the intracranial brain region: input image (a), binary mask (b), resulting intracranial brain region (c)

4.1.3. Segmentation of the lesion. Due to complexity and large variations of anatomical structures within the intracranial brain region, it is usually not possible to automatically perform accurate segmentation of the cerebrospinal fluid. Therefore, in the proposed approach, segmentation of CSF requires user interaction. In particular, the operator is expected to indicate seeds for a foreground (i.e., brain lesion due to hydrocephalus) and a background, respectively. Input data for this step are the intracranial brain region (see Fig. 5(c)). For segmentation of the CSF, the min-cut/max-flow approach proposed by Boykov and Jolly (2001) is applied. This particular method has proven to be the most effective approach in comparison with other popular segmentation algorithms tested by the authors, including region growing (Węgliński and Fabijańska, 2012a), random walk and level set (Węgliński and Fabijańska, 2012b) approaches.

The method is a graph based approach which represents an image as a weighted and undirected graph 
Table 1. Weights for $\mathrm{n}$-links and t-links.

\begin{tabular}{|c|c|c|}
\hline Edge & Weight & For \\
\hline \hline$\{p, q\}$ & $B_{\{p q\}}$ & $\{p, q\} \in N$ \\
\hline \multirow{3}{*}{$p, S$} & $\lambda R_{p}\left({ }^{\circ} b k g '\right)$ & $p \in P, p \notin O \cup B$ \\
& $K$ & $p \in O$ \\
& 0 & $p \in B$ \\
\hline \multirow{3}{*}{$p, S$} & $\lambda R_{p}\left({ }^{\circ} o b j '\right)$ & $p \in P, p \notin O \cup B$ \\
& 0 & $p \in O$ \\
& $K$ & $p \in B$ \\
\hline
\end{tabular}

$G=(V, E)$, where $V=P \cup\{S, T\}$ is a set of nodes and $E$ is a set of edges. The set $V$ consists of a subset $P$ of nodes representing pixels, and two terminal nodes $S$ and $T$, which represent the source (foreground) and the sink (background), respectively. There are also two types of undirected edges in set $E$ : n-links, which connect neighboring pixels and t-links, which connect pixels with the terminals. Each pixel has up to four n-links to the neighboring pixels and two t-links, $\{p, S\}$ and $\{p, T\}$, to the source and the sink respectively. A sample graph obtained for a $3 \times 3$ image is shown in Fig. 6
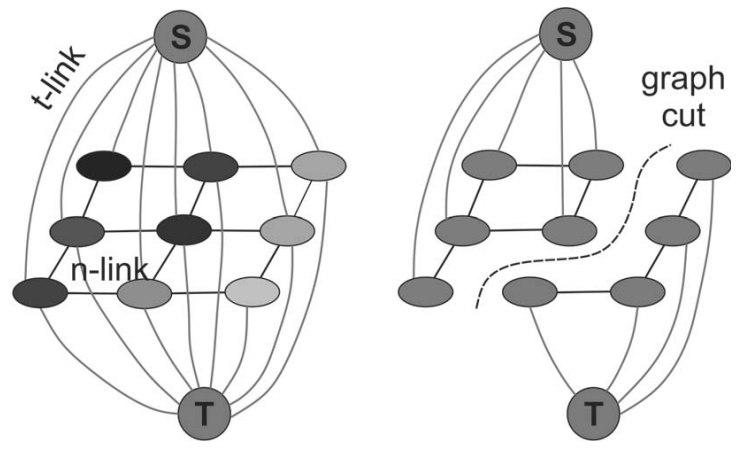

Fig. 6. Sample graph obtained for a $3 \times 3$ image.

Weights assigned to edges define their capacities. Particularly, weights $B_{\{p q\}}$ assigned to n-links describe similarities between nodes $p$ and $q$ (boundary term). The weights $R_{p}(\cdot)$ assigned to t-links define individual penalties $R_{p}\left({ }^{\prime} o b j '\right)$ and $R_{p}$ (' $\left.b k g^{\prime}\right)$ for assigning the pixel $p$ to an object $O$ and a background $B$ (the regional term). The suggested edge weights are given Table 1, where

$$
K=1+\max _{p \in P} \sum_{q:\{p, q\}} B_{\{p, q\}},
$$

and $\lambda$ is a scaling factor indicating the importance of the regional term versus the boundary term. According to min-cut/max-flow theory, in the graph $G$ optimal image segmentation is given by the edges which saturate when the maximum flow is sent from the source $S$ to the $\operatorname{sink} T$. In the proposed approach, the weights $B_{\{p q\}}$ for n-links are set in accordance with the following equation:

$$
B_{\{p, q\}}=\exp \left(-\frac{\left(I_{p}-I_{q}\right)^{2}}{2 \sigma^{2}}\right) \frac{1}{d(p, q)},
$$

where $I_{p}$ and $I_{q}$ are the intensities of neighboring pixels $p$, and $q, \sigma$ denotes the standard deviation of the intensity within the image while $d(p, q)$ is the Euclidean distance between the pixels calculated with regard to their intensities and the standard deviation of intensity within a $3 \times 3$ neighborhood.

The weights $R_{p}$ assigned to t-links are determined with regard to conditions imposed by the user on a foreground and a background, respectively. The user is supposed to indicate seeds for the object and the background on a single (manually selected) slice. This information is next used to weigh n-links in every slice. In particular, regional penalties are defined as probabilities that a pixel belongs to an object $O$ and a background $B$. For this purpose, based on seed pixels indicated by the user, the histograms of intensity in the foreground and the background are determined. The regional penalties $R_{p}\left({ }^{\prime} o b j\right.$ ') and $R_{p}\left({ }^{\prime} b k g\right.$ ') are then set as negative log-likelihoods in accordance with

$$
\begin{aligned}
& R_{p}\left({ }^{\prime} o b j '\right)=-\ln \operatorname{Pr}\left(I_{p} \mid O\right), \\
& R_{p}\left({ }^{\prime} b k g '\right)=-\ln \operatorname{Pr}\left(I_{p} \mid B\right) .
\end{aligned}
$$

Segmentation of the CSF is performed in a $2.5 \mathrm{D}$ manner. In particular, every slice is subjected to mincut/max-flow image segmentation separately, and results of 2D segmentation are next combined into a 3D binary image of the lesion. Results of CSF segmentation from a sample CT slice are presented in Fig. 7. In particular, the input slice is shown in Fig.7 (7) - light grey marks indicate seed pixels for the object while dark gray marks indicate seed pixels for background. A binary image after lesion segmentation is shown in Fig. 7(b).

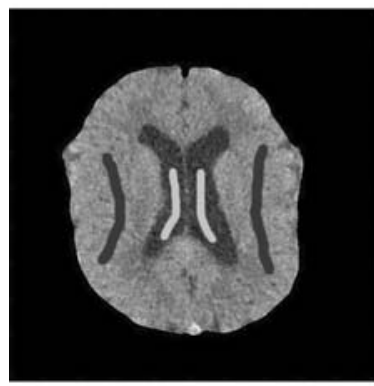

(a)

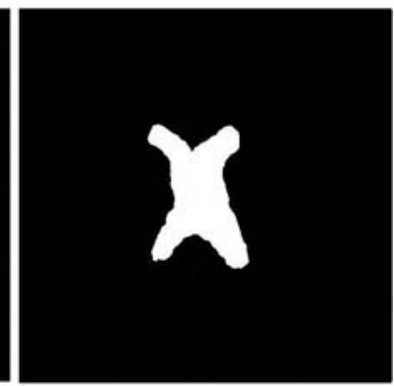

(b)
Fig. 7. CSF segmentation from the intracranial brain region: input image (a), binary image after segmentation (b).

4.2. Image analysis. Automatic determination of indices of the disease progress is performed based on binary images after segmentation. In particular, the binary image of the inner skull (intracranial brain) and the binary image of the cerebrospinal fluid are used to calculate characteristic dimensions of the brain and the lesion, respectively. 
These dimensions must be measured in a direction perpendicular to the central sagittal plane of the head. Therefore, the plane is found before a further quantitative analysis of the indices considered.

\subsubsection{Determination of the central sagittal axis of} the brain. Projection of the central sagittal plane of the head on transversal planes is the following linear function which describes the central sagittal axis of each brain slice:

$$
y=\alpha x+\beta,
$$

where $\alpha=\tan \theta, \theta$ is the orientation (angle) of the head, $\beta$ is some parameter, and $x, y$ are pixel coordinates. Parameters $\alpha$ and $\beta$ are found using a-priori knowledge about head shape. In particular, the function (12) is defined by a line which passes through the tip of the nose $\left(x_{1}, y_{1}\right)$ and the gravity center of the intracranial region $\left(x_{2}, y_{2}\right)$, as shown in Fig. 8] Calculations are performed with regard that the Cartesian coordinate system originating in the bottom left corner of a single slice. The param-

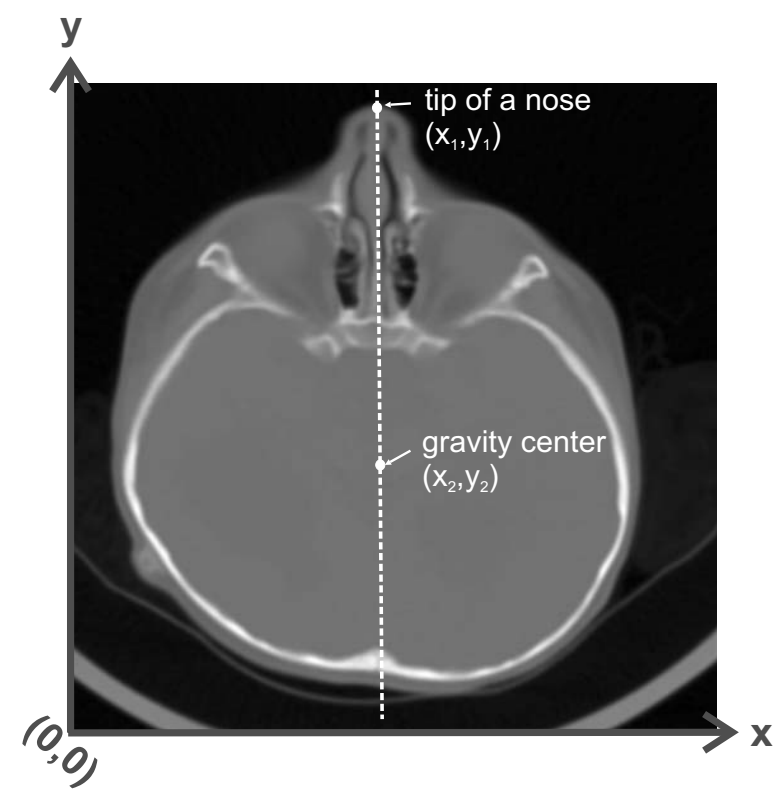

Fig. 8. Determination of the central sagittal axis of the head.

eters of the center line are

$$
\begin{gathered}
\alpha=\frac{y_{2}-y_{1}}{x_{2}-x_{1}}, \\
\beta=\frac{x_{2} y_{1}-x_{1} y_{2}}{x_{2}-x_{1}} .
\end{gathered}
$$

Determination of the central sagittal axis is performed on a binary image of the inner skull. The slice used for determination of the axis is the one with the top most "white" pixels included in the brain region. These pixels correspond to the tip of the nose and are usually found within 5-10 slices. Results of determination of the central sagittal axis for sample CT brain slices are shown in Fig. 9 .
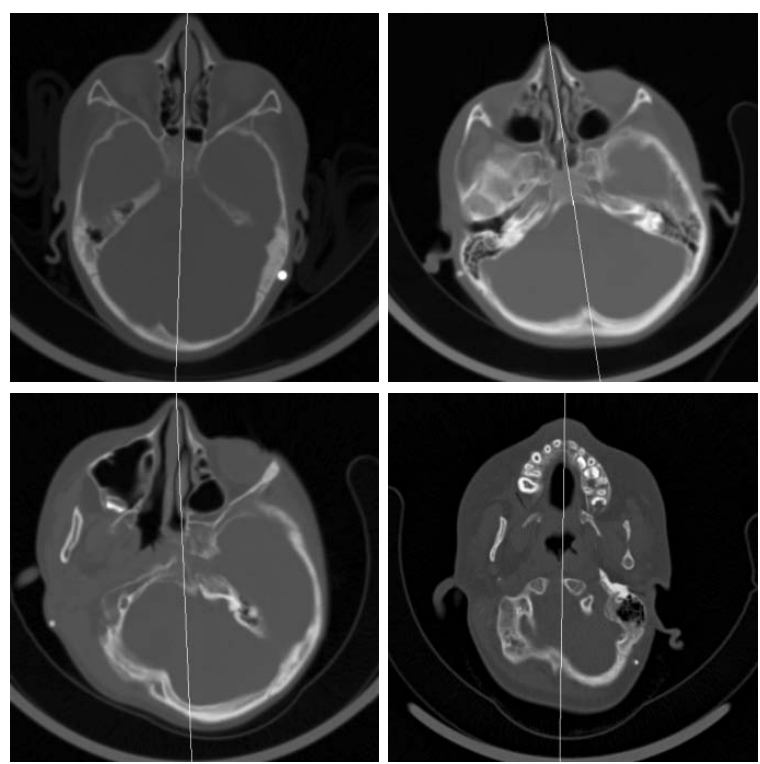

Fig. 9. Central sagittal axis of the brain determined for sample CT slices.

From Fig. 9 it can be seen that central sagittal axes were determined properly even in the case of irregular skulls deformed due to hydrocephalus.

4.2.2. Determination of the maximal width of the inner skull. For determination of the maximal width of the inner skull $\phi_{b}$, logical operations are applied. The value of $\phi_{b}$ must be measured in a direction perpendicular to the central sagittal axis of the head. Therefore, first, a family of functions perpendicular to the axis is found using

$$
y=-\alpha^{-1} x+\gamma_{i} .
$$

Next, these functions are plotted within the image domain, resulting with binary images $q_{\gamma_{i}}$ defined by

$$
q_{\gamma_{i}}(x, y)= \begin{cases}1 & \text { if } \left.y=\left\lceil-\alpha^{-1} x+\gamma_{i}\right\rceil\right\} \\ 0 & \text { otherwise }\end{cases}
$$

where $i \in\{0,1, \ldots, M\}$ and $M$ is the height of a single slice.

Finally, the logical operator AND is evaluated between the regarded brain slice $b_{k}$ and images $q_{\gamma_{i}}$ in order to find consecutive chords $\phi_{b i}$ of the brain (see Fig. (10)). The maximal width of the inner skull $\phi_{b_{\max }}$ is equal to the longest chord, whose length is given by

$$
\phi_{b_{\max }}=\frac{\max _{\gamma_{i}}\left(\sum b_{k}(x, y) \cap q_{\gamma_{i}}(x, y)\right)}{\cos \psi},
$$




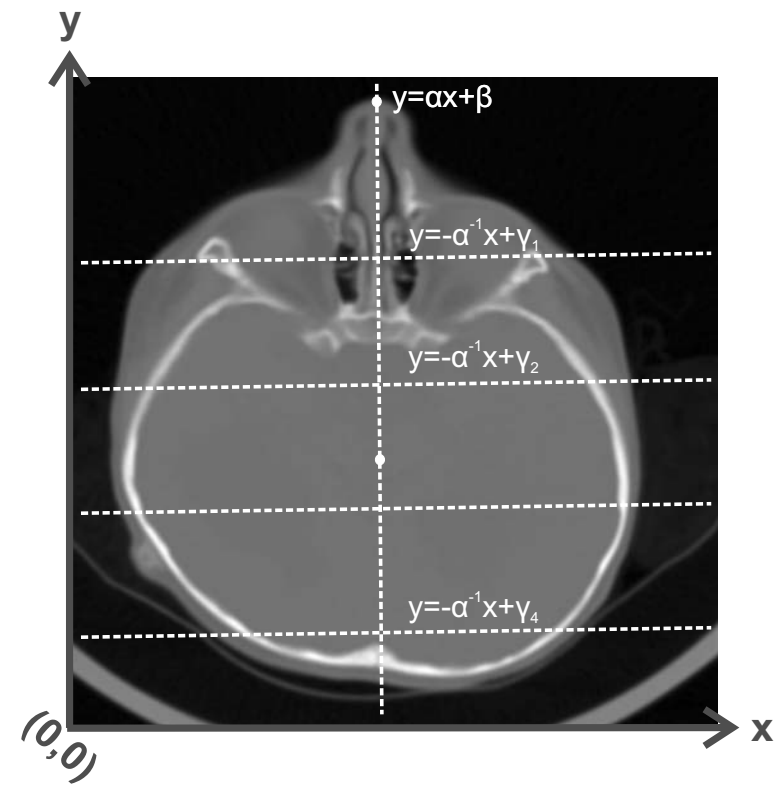

Fig. 10. Determination of the maximal width of the inner skull.

where $\psi=\operatorname{atan}\left(-\alpha^{-1}\right)$.

Results of determination of the maximal width of the inner skull in sample CT brain slices are shown in Fig. 11. The horizontal line shows the maximal width of the inner skull in a given slice while the vertical line shows the central sagittal axis of the head.
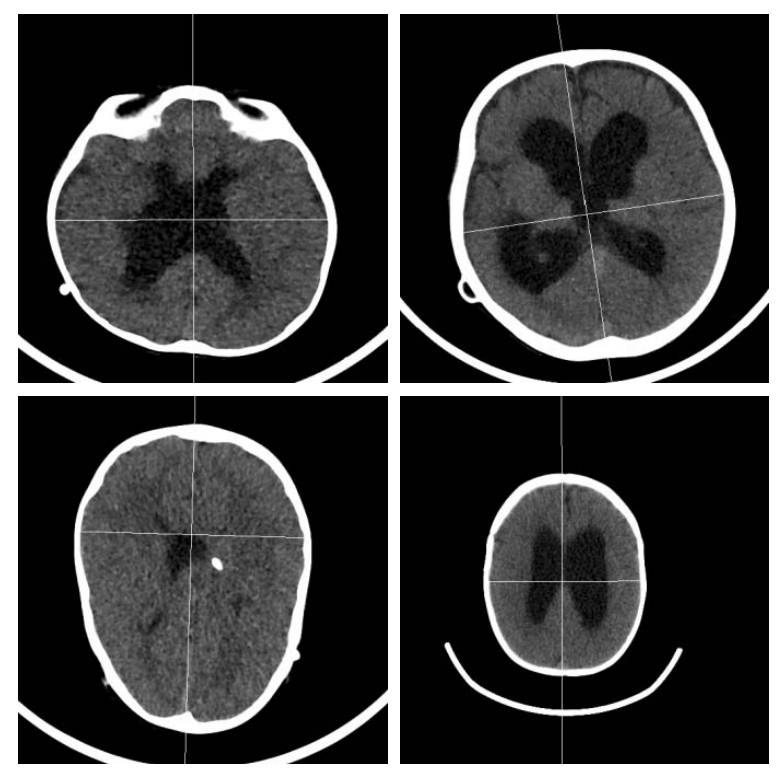

Fig. 11. Determination of the maximal width of the inner skull in sample CT brain slices.

4.2.3. Determination of the maximal width of the anterior and the occipital horns. The maximal width of the anterior horns $d_{f h}$ and the maximal width of the oc- cipital horns $d_{o h}$ are found based on a binary image of the cerebrospinal fluid. In particular, the most representative slice $c_{k}$ is used. The slice is selected manually. Both $d_{f h}$ and $d_{o h}$ are defined as the maximal distance between two pixels which belong to both a line $y=-\alpha^{-1} x+\gamma_{i}$ perpendicular to the central sagittal axis of the head and the binary region of the CSF in slice $c_{k}$. However, in the case of the maximal width of the anterior horns, $\gamma_{i}>\gamma$, while in the case of the maximal width of the occipital horns, $\gamma_{i}<\gamma$, where $\gamma$ is the parameter of a linear function $y=-\alpha^{-1} x+\gamma$ containing the maximal width of the inner skull. The values of $d_{f h}$ and $d_{o h}$ can be respectively defined by

$$
\begin{aligned}
d_{f h} & =\max _{\gamma_{i}>\gamma} d\left(\mathbf{x}_{1}, \mathbf{x}_{2}\right), \\
d_{o h} & =\max _{\gamma_{i}<\gamma} d\left(\mathbf{x}_{1}, \mathbf{x}_{2}\right),
\end{aligned}
$$

where

$$
\mathbf{x}=\left\{(x, y): y=\left\lceil-\alpha^{-1} x+\gamma_{i}\right\rceil \text { and } c_{k}(x, y)=1\right\}
$$

and $d$ stands for the Euclidean distance regarding pixel dimensions given in DICOM tags Pixel Spacing $X$ and Pixel Spacing $Y$ (DICOM, 2004). The procedure of determination of the maximal width of the anterior and the occipital horns is shown in Fig. 12

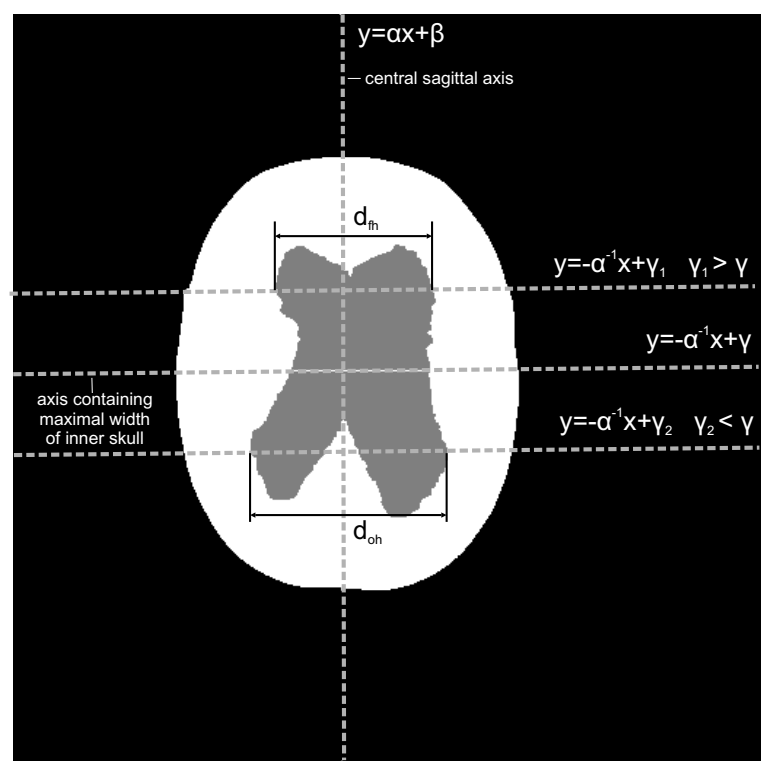

Fig. 12. Determination of the maximal width of the anterior horns $\left(d_{f h}\right)$ and of the occipital horns $\left(d_{o h}\right)$.

Results of determination of the maximal width of the anterior horns $\left(d_{f h}\right)$ and the maximal width of the occipital horns $\left(d_{o h}\right)$ in sample CT brain slices are shown in Fig. 13. The vertical line shows the central sagittal axis of the head while the maximal widths of the horns are indicated by the horizontal lines. Additionally, the region of cerebrospinal fluid is outlined. 


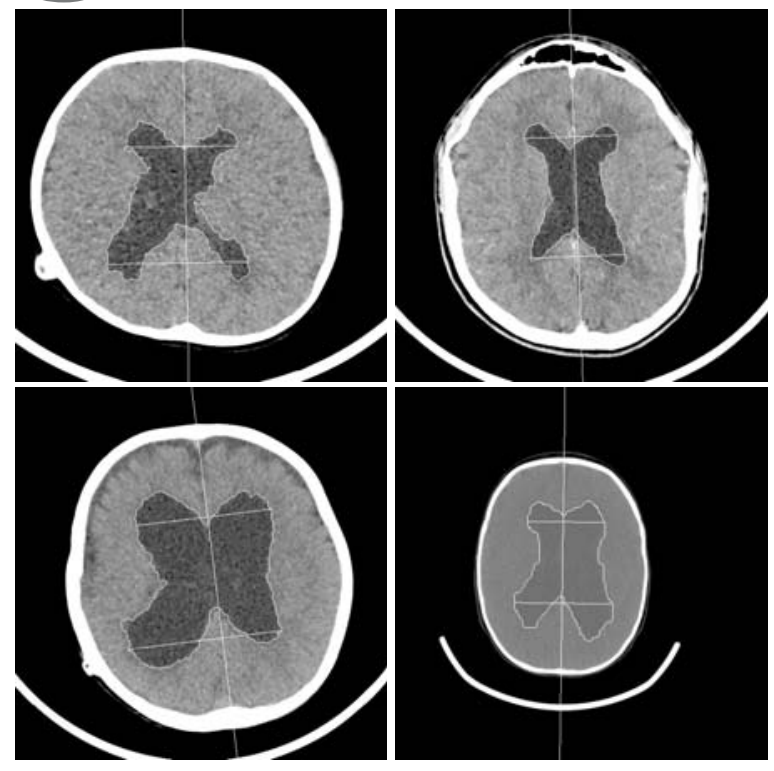

Fig. 13. Determination of the maximal width of the anterior and the occipital horns in sample CT slices.

4.2.4. Determination of the skull width at the level of the CSF minimal width. In this step the minimal width of the cerebrospinal fluid is first found. The procedure is quite like in the case of determination of the maximal width of the skull. In particular, the minimal width of the CSF is the minimal distance between two pixels which belong to both the line $y=-\alpha^{-1} x+\gamma_{i}$ perpendicular to the central sagittal axis of the head and the outline of the binary region of CSF. Additionally, the distance is computed only if the line $y=-\alpha^{-1} x+\gamma_{i}$ crosses the border exactly in two pixels, has one common pixel with the central sagittal axis of the head and is located above the maximal width of the occipital horn but below the maximal width of the frontal horn (see Fig. 12).

After the minimal width of the CSF has been found, the corresponding width of the skull is determined in accordance with

$$
\phi_{b}=\frac{\sum b_{k}(x, y) \cap q_{\gamma_{\min }}(x, y)}{\cos \psi}
$$

where $y=-\alpha^{-1} x+\gamma_{\min }$ is a line containing the minimal width of the CSF, $q_{\gamma}$ is the binary image given by Eqn. (17) and $\psi=\operatorname{atan}\left(-\alpha^{-1}\right)$.

Results of determination of the skull width at the level of the minimal width of CSF in sample CT brain slices are shown in Fig. 14, The vertical line shows the central sagittal axis of the head while the skull width is indicated by the horizontal line. Additionally, the region of the cerebrospinal fluid is outlined.

After determination of the maximal width of the inner skull $\phi_{b_{\max }}$, the maximal width of the anterior horns $d_{f h}$, the maximal width of the occipital horns $d_{o h}$ and the

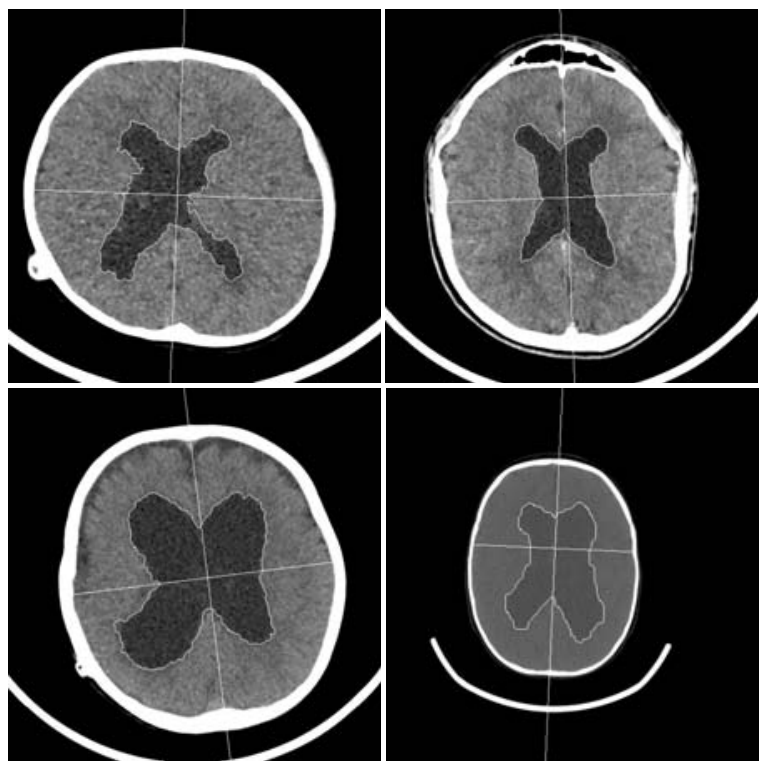

Fig. 14. Results of determination of the skull width at the level of the minimal CSF width.

skull width at the level of the CSF minimal width $\phi_{b}$, the Evans ratio as well as the frontal and occipital horn ratio can be easily calculated from Eqns. (1) and (2), respectively.

4.2.5. Determination of the frontal horn radius. The frontal horn radius is found based on a binary image of the cerebrospinal fluid. Since the FHR is defined as the widest diameter of the frontal horns, the selected range of slices of a binary image of the CSF is analyzed sliceby-slice to find the maximal value of the radius. Slices to be analyzed are indicated manually. The proposed approach determines the radius of the left $\left(\mathrm{FHR}_{L}\right)$ and the right $\left(\mathrm{FHR}_{R}\right)$ horn separately.

For every slice, a skeleton of the CSF region is first found using iterative thinning described by Gonzalez and Woods (2007). Branches of the skeleton corresponding to the frontal horns are next separated. These can be easily found as they start from the top leftmost and the top rightmost endpoints of the skeleton, and end at the top-most branchpoint (see Fig. 15). The coordinates of branches corresponding to horns are next used to find equations of long axes of the left and the right horn. In particular, the axes are approximated by linear functions $y=p_{i} x+q_{L}$ and $y=p_{i} x+q_{R}$, determined using the least-squares algorithm.

Next, the left and the right horn are separated from the current slice. In particular, the slice is first divided along the central sagittal axis into the left and the right part (see Fig.16(a)). Then the minimal width of every part is found above the level of the maximal skull width (see Fig. 16(b)). Images $h_{L}(x, y)$ and $h_{R}(x, y)$ of the left and 


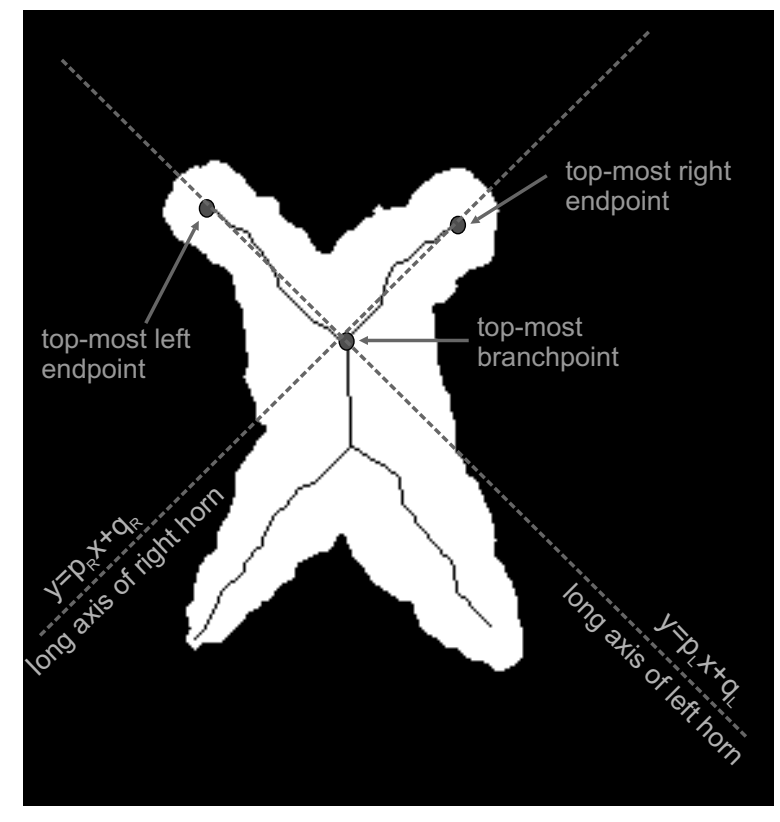

Fig. 15. Determination of long axes of the frontal horns.

right horns are binary regions located above the minimal widths (see Fig. 16 (c) and (d)).

After the horns have been separated, both radii are determined as a the maximal distance between two hornpixels which belong to the line perpendicular to the horn long axis (see Fig. 17). In particular, $\mathrm{FHR}_{L}$ and $\mathrm{FHR}_{R}$ are defined as

$$
\mathrm{FHR}_{L}=\max _{s_{L i}} d\left(\mathbf{x}_{1}, \mathbf{x}_{2}\right),
$$

where $\mathbf{x}=\left\{(x, y): y=\left\lceil-p_{L}^{-1} x+s_{L i}\right\rceil\right.$ and $h_{L}(x, y)=$ $1\}$,

$$
\mathrm{FHR}_{R}=\max _{s_{R i}} d\left(\mathbf{x}_{1}, \mathbf{x}_{2}\right),
$$

where $\mathbf{x}=\left\{(x, y): y=\left\lceil-p_{R}^{-1} x+s_{R i}\right\rceil\right.$ and $h_{R}(x, y)=$ $1\}$,

and $d$ stands for the Euclidean distance determined with regard to pixel dimensions given in DICOM tags Pixel Spacing $X$ and Pixel Spacing $Y$ (DICOM, 2004).

Finally, the frontal horn radius is defined by the following equation:

$$
\mathrm{FHR}=\max \left(\mathrm{FHR}_{R}, \mathrm{FHR}_{L}\right) .
$$

Results of frontal horn radius determination in sample CT slices are shown in Fig. 18, For every horn, the long axis and the maximal width are shown.

4.2.6. Determination of the ventricular angle. For determination of the ventricular angle, images $h_{L}(x, y)$

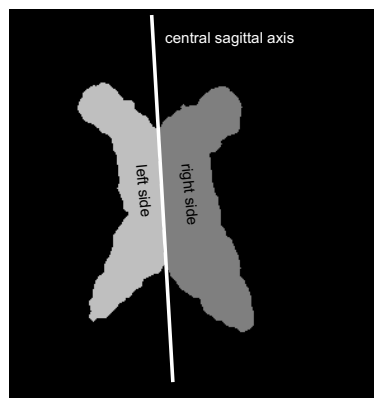

(a)

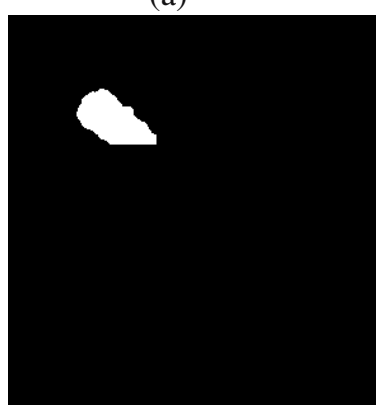

(c)

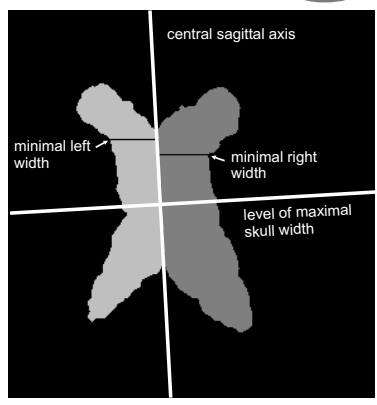

(b)

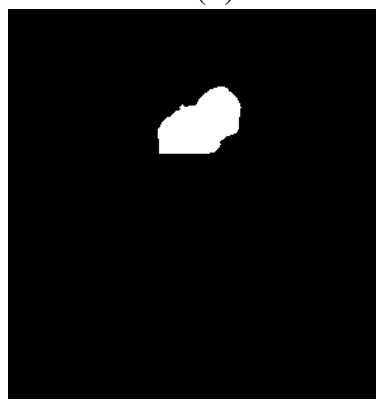

(d)
Fig. 16. Separation of horns: image of the CSF divided into the left and the right part (a), minimal width of the left and the right part (b), left horn (c), right horn (d).

and $h_{R}(x, y)$ of frontal horns are used again. In particular, the ventricular angle is found for the horn with a higher frontal horn radius. The value of the VA is determined as the angle between the central sagittal axis of the head and a line fitted into the upper edge of a horn, as shown in Fig. 19

In order to find the upper edge of a horn, its outline is first found using the Sobel gradient operator (Gonzalez and Woods, 2007) (see Fig. 20(b)). Next, among the remaining pixels, a set of consecutive pixels composing the longest, monotonic slope (non-increasing for $h_{L}(x, y)$ and non-decreasing for $h_{R}(x, y)$ ) is found (see Fig. 20(c)). These pixels are used to fit the line $y=m x+n$ which approximates the upper edge of the horn (see Fig. 20(d)). The values of $m$ and $n$ are found using the least-squares fitting algorithm.

After the equation of a line approximating the upper edge of a horn has been found, the ventricular angle is calculated using

$$
\mathrm{VA}=\operatorname{atan}\left(\left|\frac{a-m}{1+a m}\right|\right)
$$

where $a$ and $m$ are the slope of the central sagittal axis of the head and the line fitted into the upper edge of the horn, respectively. 


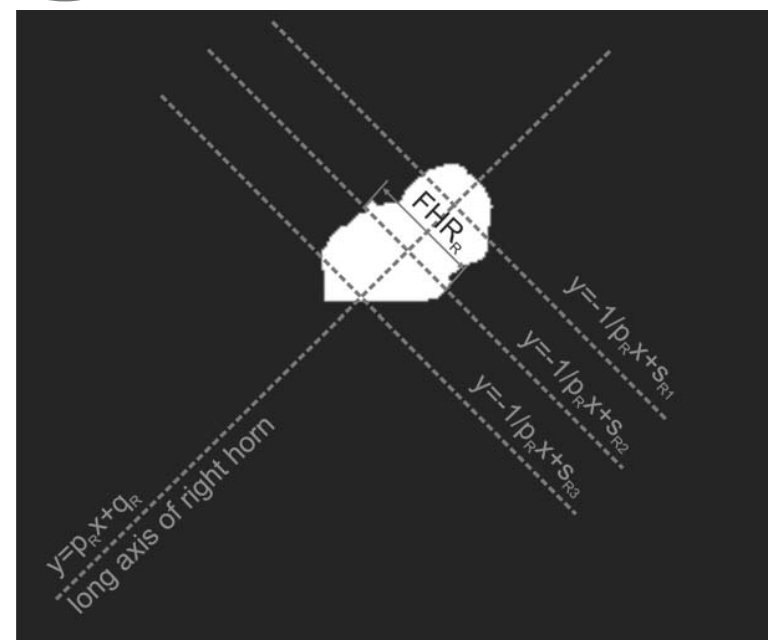

Fig. 17. Determination of the maximal width of the frontal horn.
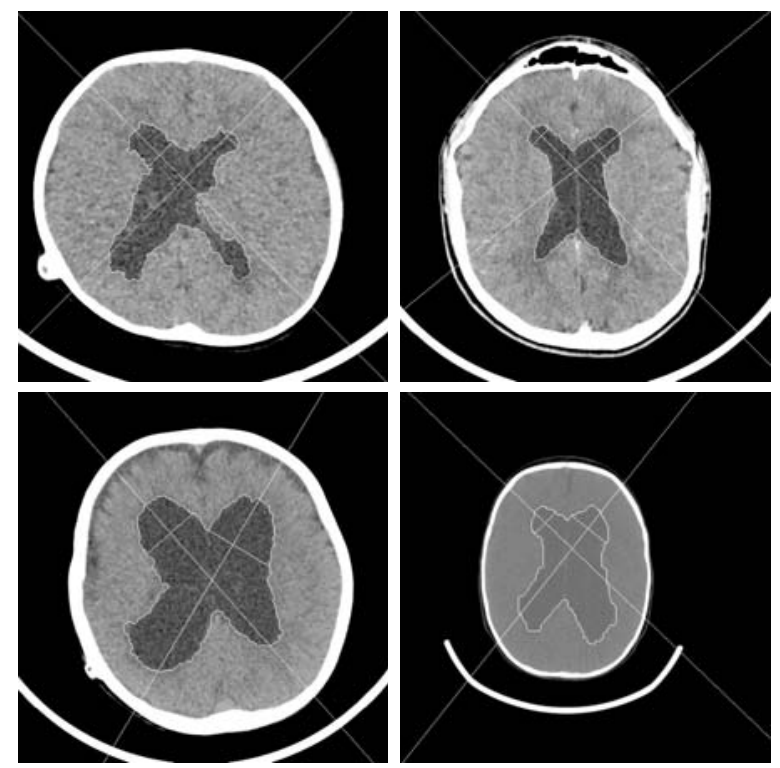

Fig. 18. Determination of the frontal horn radius.

\section{Results and validation}

This section presents results of applying the introduced image processing and analysis algorithms to the numerical assessment of hydrocephalus in 10 sample CT examinations (Cases 1-10). Each examination of a patient was with an enlarged ventricular system. Examinations consisted of 20 to 100 grayscale slices representing consecutive cross sections of the brain. All slices were stored in the DICOM format and coded in 16-bit integers. Spatial resolution of a single slice was $512 \times 512$ pixels. All introduced algorithms were implemented in MATLAB 2012b. For min-cut/max-flow segmentation, a C++ source code provided by the authors of the method was used and included into the project as MEX functions. The code is available at http://vision.csd. uwo.ca/code

For each case, indices of the disease progress (i.e.,

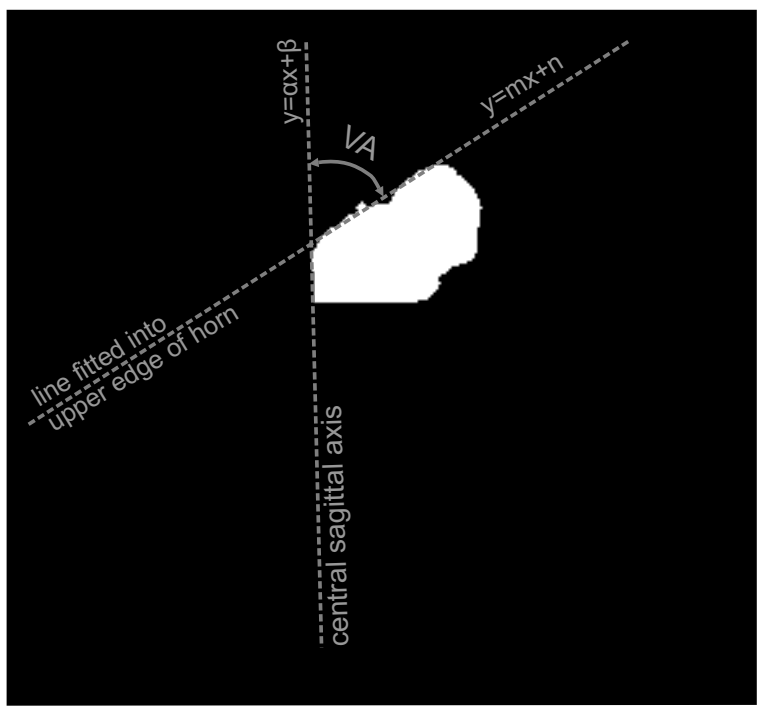

Fig. 19. Determination of the ventricular angle.

ER, FOHR, VA, FHR) were determined automatically using the proposed approach and compared with those of manual assessment performed by both a radiologist from the Polish Mother's Memorial Hospital, Research Institute in Łódź, and a trained operator. In order to determine the regarded indices, the radiologist used hospital equipment while the trained operator used Amira software. All measurements were performed on the most representative slices indicated by a radiologist.

Results obtained using the above mentioned procedure for 10 sample cases are shown in Figs. 21, 24 In particular, Fig. 21 presents results of the Evans ratio determination. In Fig. 22 the determined values of frontal and occipital horn ratios are shown. Measurements of the ventricular angle are summarized in Fig.23, while Fig. 24 shows the values of a frontal horn radius. In every figure, the series auto refers to results provided by the proposed approach, the series radiol corresponds to measurements performed by a radiologist, while the results obtained by a trained operator are represented by the series oper. The values of the FHR are given in milimeters while those of the VA in degrees.

The corresponding measurement errors are summarized in Table 2. The values determined by a radiologist were used as a reference. Next, both the relative errors between the values obtained by the proposed approach and the radiologist (the series auto/radiol) as well as values measured by the trained operator and the radiologist (the series oper/radiol) were determined for all the indices considered. The case ID is indicated at the beginning of each row. Additionally, the last row presents the average measurement errors obtained for every parameter.

Results presented in Figs. 21, 24 show that, in most cases, the values of indices determined automatically us- 
Table 2. Relative errors of the determined indices of the hydrocephalus progress.

\begin{tabular}{|c|c|c|c|c|c|c|c|c|}
\hline Case ID & \multicolumn{2}{|c|}{ ER } & \multicolumn{2}{c|}{ FOHR } & \multicolumn{2}{c|}{ VA } & \multicolumn{2}{c|}{ FHR } \\
\hline & auto/radiol & oper/radiol & auto/radiol & oper/radiol & auto/radiol & oper/radiol & auto/radiol & oper/radiol \\
\hline \hline 1 & $19.57 \%$ & $10.87 \%$ & $5.08 \%$ & $5.08 \%$ & $8.13 \%$ & $2.15 \%$ & $3.07 \%$ & $10.25 \%$ \\
\hline 2 & $6.25 \%$ & $6.25 \%$ & $12.50 \%$ & $1.79 \%$ & $8.58 \%$ & $14.06 \%$ & $2.48 \%$ & $10.61 \%$ \\
\hline 3 & $12.00 \%$ & $0.00 \%$ & $7.27 \%$ & $1.82 \%$ & $9.17 \%$ & $12.10 \%$ & $10.24 \%$ & $17.35 \%$ \\
\hline 4 & $30.30 \%$ & $6.06 \%$ & $7.14 \%$ & $2.38 \%$ & $3.55 \%$ & $1.56 \%$ & $11.54 \%$ & $3.66 \%$ \\
\hline 5 & $21.88 \%$ & $3.13 \%$ & $22.22 \%$ & $5.56 \%$ & $7.79 \%$ & $9.04 \%$ & $43.97 \%$ & $10.64 \%$ \\
\hline 6 & $56.41 \%$ & $56.41 \%$ & $1.96 \%$ & $3.92 \%$ & $2.65 \%$ & $4.56 \%$ & $10.09 \%$ & $1.19 \%$ \\
\hline 7 & $28.13 \%$ & $0.00 \%$ & $4.65 \%$ & $6.98 \%$ & $9.50 \%$ & $5.44 \%$ & $21.44 \%$ & $12.01 \%$ \\
\hline 8 & $14.06 \%$ & $18.75 \%$ & $4.11 \%$ & $2.74 \%$ & $3.15 \%$ & $15.36 \%$ & $2.62 \%$ & $16.34 \%$ \\
\hline 9 & $13.95 \%$ & $6.98 \%$ & $1.89 \%$ & $3.77 \%$ & $11.80 \%$ & $3.00 \%$ & $5.67 \%$ & $3.99 \%$ \\
\hline 10 & $21.21 \%$ & $3.03 \%$ & $4.35 \%$ & $0.00 \%$ & $4.29 \%$ & $8.07 \%$ & $6.46 \%$ & $6.98 \%$ \\
\hline Avg. & $22.38 \%$ & $11.15 \%$ & $7.12 \%$ & $3.40 \%$ & $6.86 \%$ & $7.53 \%$ & $11.76 \%$ & $9.30 \%$ \\
\hline
\end{tabular}

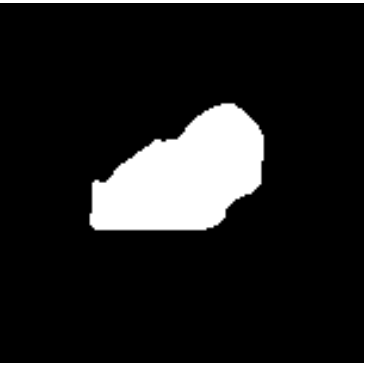

(a)

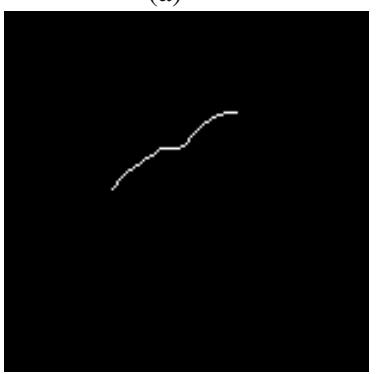

(c)

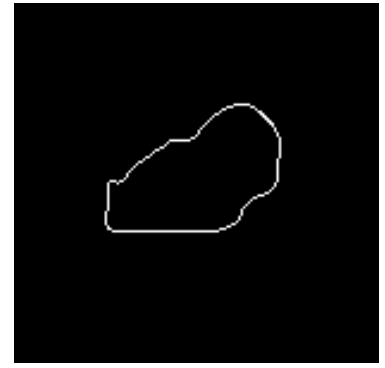

(b)

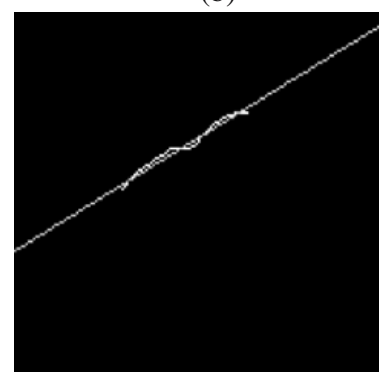

(d)
Fig. 20. Consecutive steps of approximation of the upper edge of the frontal horn with a linear function: frontal horn (a), edge of a frontal horn (b), longest non-decreasing slope (c), line fitted into the upper edge of the horn (d).

ing the proposed approach are close to those determined manually by both the radiologist and the trained operator. The highest disparities between the automatic and the manual measurements appear in the case of the Evans ratio, where in more than half of cases the disparity is higher than $15 \%$. However, in the case of the ER index, the average disparity between the measurements performed by a radiologist and an operator is over $11 \%$, which suggests that even a trained operator was not able to repeat manual measurements. The average errors of automatic determination of the remaining indices, i.e., FOHR, VA and FHR, are less than $10 \%$. However, it should be also underlined that these statistics are worsened by Case 5, where image

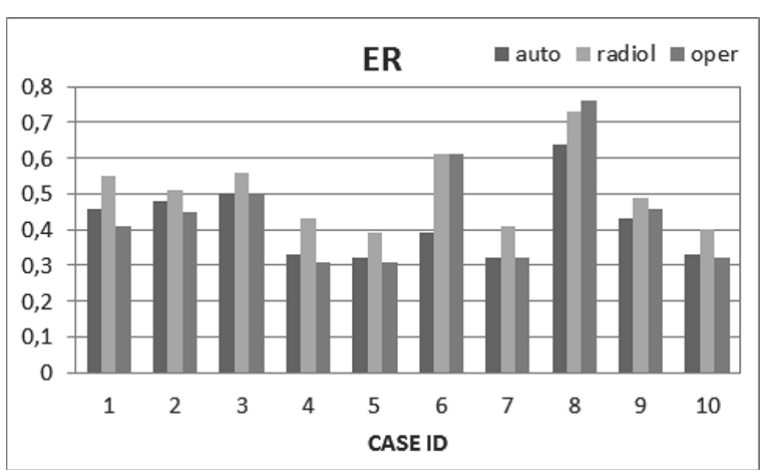

Fig. 21. Determined values of the Evans ratio.

segmentation failed (see Fig. 25). In this case only the radiologist was able to properly measure the regarded indices. This resulted in a significant disparity (even 56\%) between the manual and the automatic measurements in the case of all regarded indices. This case also shows that even in automatic systems the supervision and verification by a specialist are necessary.

In most cases, disparities between the determined values of the ER, FOHR and FHR are caused by slight differences in selection of characteristic dimensions by the automatic and the manual method. In particular, the proposed automatic approach ensures that the characteristic dimensions are measured in parallel planes, perpendicular to the central sagittal axis of the head. This is the only way to measure and designate true characteristic dimensions. In the case of manual measurements, this condition is not always fulfilled and the dimensions may be measured at a different angle to the central sagittal axis. This introduces measurement errors into the ER, FHR and FOHR indices.

Lastly, it should be emphasized that the comparison shown in Figs. 21, 24 and Table 2 is very tentative as manual measurements performed by a radiologist are subjective and cannot be regarded as a ground truth. Furthermore, the differences between values of indices calculated 


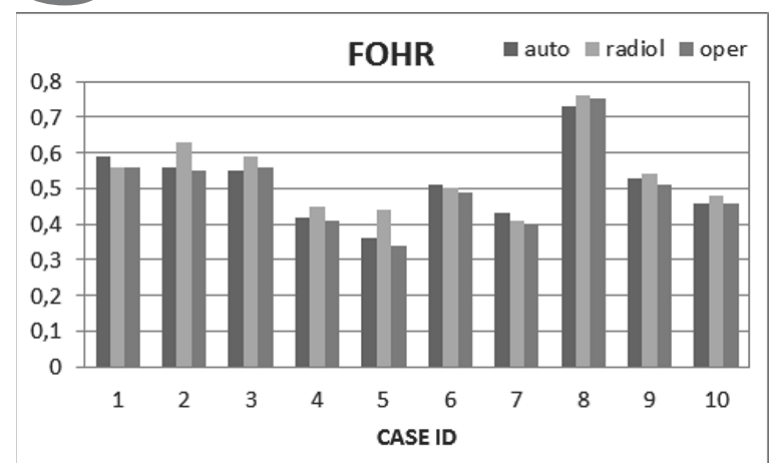

Fig. 22. Determined values of the frontal and the occipital horn ratio.

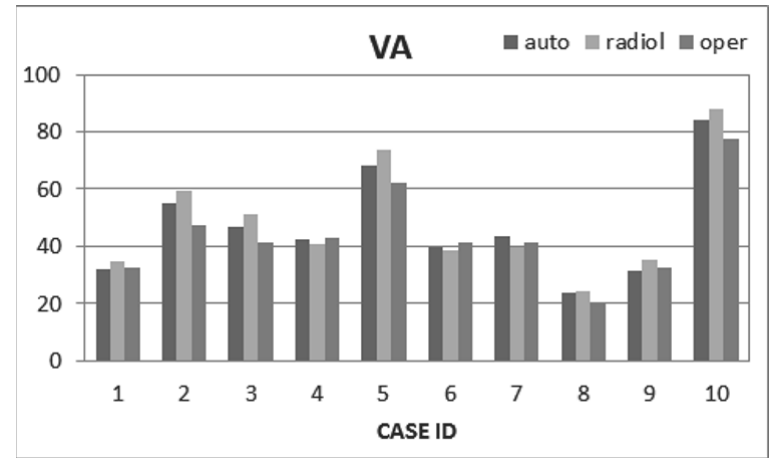

Fig. 23. Determined values of the ventricular angle.

by a radiologist and a trained operator prove the subjectivity of the manual measurements. As a result, it is not possible to determine the exact accuracy of the introduced approach. It is also very difficult to judge which results are most accurate. However, despite this uncertainty, the physicians from the Polish Mother's Memorial Hospital in Łódź confirmed the potential usefulness of the introduced image processing and analysis algorithms in their clinical routine.

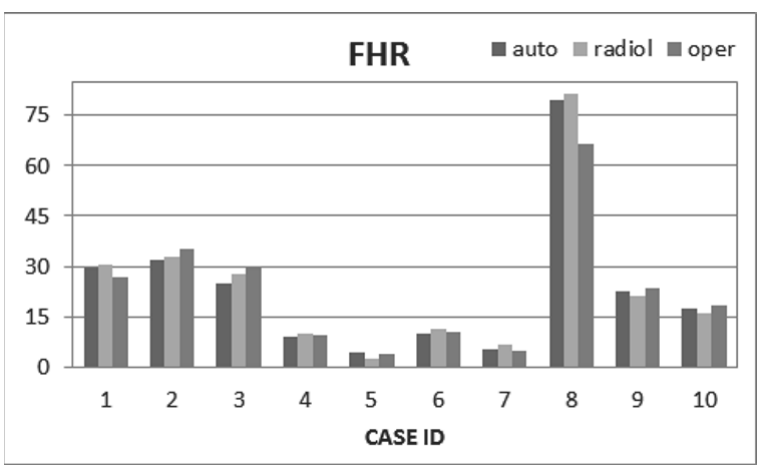

Fig. 24. Determined values of the frontal horn radius.

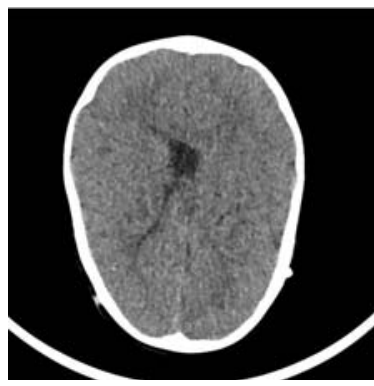

(a)

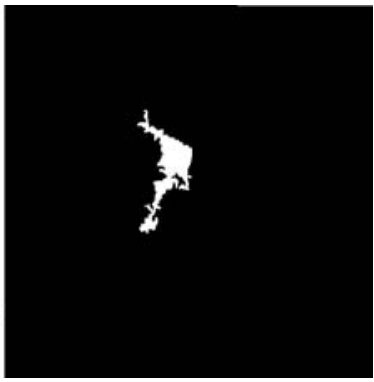

(b)
Fig. 25. Incorrect segmentation in Case 5: original image (a), segmentation result (b).

\section{Conclusions}

Accurate detection and analysis of hydrocephalus has great importance for today's neurosurgery. These steps are necessary to make the right decision regarding open surgery and postoperative control of the patient. Besides a visual assessment of hydrocephalus based on CT or MRI scans, additional calculations of the characteristic ratios often allow diagnosing whether the disease process is active. However, recent measurement methods are performed mainly manually by a radiologist. Therefore, such calculations are subjective, cumbersome and often tentative.

In the research described in this paper, image processing and analysis algorithms were applied to support objective diagnosis of hydrocephalus in children. Particularly, methods for segmentation of significant brain regions and calculation of popular indices of the disease progress were sketched. This concept forms a basis for the development of an application for automatic detection and analysis of hydrocephalus in CT examinations. Quantitative comparison between the results obtained by the introduced approach and manually conducted measurements shows that the work is going in the right direction. In particular, results of manual and automatic determination of disease indices are similar, which verifies the proposed algorithms and indicates their reliability.

Methods described in this paper are a universal set of tools for supporting radiologists in their everyday practice on the assessment of hydrocephalus in children. Introduction of these methods into the clinical routine may increase objectivity and repeatability of the assessment of the hydrocephalus progress.

The introduced set of image processing and analysis algorithms is still affected by a 'human factor'. In particular, it requires a radiologist to manually indicate the most representative slices used for automatic determination of indicators of the disease progress. Therefore, future work will be on automatic selection of the characteristic slices. Research will also be carried out on establishing the relationship between the regarded indices 
of the hydrocephalus progress and volumetric indicators of the disease proposed in our previous paper (Węgliński and Fabijańska, 2012a).

Recently, the total processing time of the proposed algorithm (including all the necessary processing and calculation steps) is about 2.7 seconds per slice (i7-3612QM CPU@2.10GHz, 16 GB RAM). Therefore, additional work will be performed to optimize and speed up the introduced approach. It will also be rebuilt into the $\mathrm{C}++$ environment under Windows.

\section{Acknowledgment}

This research was funded by the Ministry of Science and Higher Education of Poland from funds for science in the years 2013-2015 in the framework of the Iuventus Plus programme (project no. IP 2012011272 ).

\section{References}

Ambarki, K., Wahlin, A., Birgander, R., Eklund, A. and Malm, J., (2011). MR imaging of brain volumes: Evaluation of a fully automatic software, American Journal of Neuroradiology 32(2): 408-412

Barkovich, A.J., (2005). Pediatric Neuroimaging, Lippincott Williams \& Wilkins, New York, NY.

Bosnjak, A., Montilla, G., Villegas, R. and Jara, I. (2007). 3D segmentation with an application of level set-method using MRI volumes for image guided surgery, Proceedings of the 29th Annual International Conference of the IEEE Engineering and Medicine in Biology Society, Osaka, Japan, pp. 5263-5266.

Boykov, Y. and Jolly, M.P. (2001). Interactive graph cuts for optimal boundary \& region segmentation of objects in N-D images, Proceedings of the International Conference on Computer Vision, Vancouver, Canada, Vol. 1, pp. 105-112

Butman, J.A. and Linguraru, M.G. (2008). Assessment of ventricle volume from serial MRI scans in communicating hydrocephalus, Proceedings of the 5th IEEE International Symposium on Biomedical Imaging: From Nano to Macro, Paris, France, pp. 49-52.

DICOM (n.d.). DICOM specification, http://medical.nema.org/dicom/2004.html.

Frąckiewicz, M. and Palus, H. (2011). KHM clustering technique as a segmentation method for endoscopic colour images, International Journal of Applied Mathematics and Computer Science 21(1): 203-209, DOI: 10.2478/v10006011-0015-0.

Gonzalez, R.C. and Woods, R.E. (2007). Digital Image Processing, 3rd Edition, Prentice Hall, Englewood Cliffs, NJ.

Gupta, V., Ambrosius, W., Qian, G., Blazejewska, A., Kazmierski, R., Urbanik, A. and Nowinski, W.L. (2010). Automatic segmentation of cerebrospinal fluid, white and gray matter in unenhanced computed tomography images, Academic Radiology 17(11): 1350-1358.
Halberstadt, W. and Douglas, T.S. (2005) Fuzzy clustering of $\mathrm{CT}$ images for the measurement of hydrocephalus associated with tuberculous meningitis, Proceedings of the Annual International Conference of the IEEE on Engineering in Medicine and Biology, Shanghai, China, pp. 4014-4016.

Hamano, K., Iwasaki, N., Takeya, T. and Takita, H. (1993). A comparative study of linear measurements of the brain and three-dimensional measurement of brain volume using CT scans, Pediatric Radiology 23(3): 165-168.

Hiraoka, K., Yamasaki, H., Takagi, M., Saito, M., Nishio, Y., Iizuka, O., Kanno, S., Kikuchi, H., Kondo, T. and Mori, E. (2010). Changes in the volumes of the brain and cerebrospinal fluid spaces after shunt surgery in idiopathic normal-pressure hydrocephalus, Journal of the Neurological Sciences 296(1): 7-12.

Kanayama, S.A., Calderon, A.B., Makita, J.I.C., Ohara, Y.D., Tsunoda, A.D. and Sato, K.D. (1998). Evaluation of noninvasive cerebrospinal fluid volume measurement method with 3D-FASE MRI, Systems and Computers in Japan 29(14): 41-49.

Kulczycki, P. and Charytanowicz, M. (2010). A complete gradient clustering algorithm formed with kernel estimators, International Journal of Applied Mathematics and Computer Science 20(1): 123-134, DOI: 10.2478/v10006-010-00093.

Lie, W.-N., Peng, W.-H. and Chuung, C.-H. (2002). Efficient content-based CT brain image retrieval by using region shape features, Proceedings of the IEEE International Symposium on Circuits and Systems, Phoenix-Scottsdale, AZ, USA, Vol. 4, pp. 157-160.

Luo, F., Evans, J.W., Linney, N.C., Schmidt, M.H. and Gregson, P.H. (2010). Wavelet-based image registration and segmentation framework for the quantitative evaluation of hydrocephalus, Journal of Biomedical Imaging (2010): 1-12, Article ID: 248393.

O'Hayon, B.B., Drake, J.M., Ossip, M.G., Tuli, S. and Clarke, M. (1998). Frontal and occipital horn ratio: A linear estimate of ventricular size for multiple imaging modalities in pediatric hydrocephalus, Pediatric Neurosurgery 29(5): 245-249.

Pustkova, R., Kutalek, F., Penhaker, M. and Novak, V. (2010). Measurement and calculation of cerebrospinal fluid in proportion to the skull, Proceedings of the 9th RoEduNet IEEE International Conference, Sibiu, Romania, Vol. 8, pp. 9599.

Ruttimann, U.E., Joyce, E.M., Rio, D.E. and Eckardt, M.J. (1993). Fully automated segmentation of cerebrospinal fluid in computed tomography, Psychiatry Research: Neuroimaging 50(2): 101-119.

Schnack, H.G., Hulshoff Pol, H.E., Baare, W.F.C., Viergever, M.A. and Kahn, R.S. (2001). Automatic segmentation of the ventricular system from MR images of the human brain, NeuroImage 14(2): 95-104.

Synek, V., Reuben, J.R. and Du Boulay, G.H. (1976). Comparing Evans index and computerized axial tomography in assessing relationship of ventricular size to brain size, Neurology 26(3): 231-233. 
Węgliński, T. and Fabijańska, A. (2012). Min-cut/max-flow segmentation of hydrocephalus in children from CT datasets, Proceedings of the IEEE International Conference on Signals and Electronic Systems, Wroctaw, Poland, pp. 1-6

Węgliński, T. and Fabijańska, A. (2012) Survey over modern image segmentation algorithms on CT scans of hydrocephalic brains, Image Processing and Communications 17(4): 223-230.

Zang, X., Wang, Y., Yang, J. and Liu, Y. (2010) A novel method of CT brain images segmentation, Proceedings of the International Conference on Medical Image Analysis and Clinical Application, Wuhan, China, pp. 109-112.

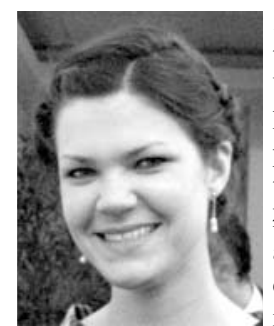

Anna Fabijańska is an assistant professor at the Institute of Applied Computer Science, Łódź University of Technology (Poland). She received her M.Sc., Ph.D. and D.Sc. degrees in computer science from the Faculty of Electrical, Electronic, Computer and Control Engineering of the Łódź University of Technology in 2006, 2007 and 2013, respectively. Her research interests include computer engineering, computer measurement systems, as well as the development of image processing and analysis algorithms for industrial and biomedical applications. She is the author or a co-author of almost 80 scientific publications. So far, she has been the manager in two and a principal investigator in three research projects financed by the Polish Ministry of Science and Higher Education and the European Union.

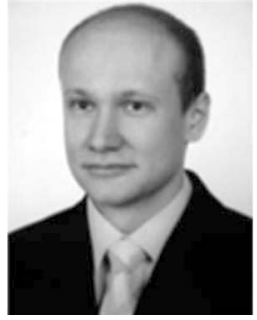

Tomasz Wegliński is a Ph.D. student at the Institute of Applied Computer Science, Łódź University of Technology (Poland). He received his M.Sc. in computer science from the Faculty of Electrical, Electronic, Computer and Control Engineering of the Łódź University of Technology in 2010. His research interests include computer engineering, as well as the development of image processing and analysis algorithms for biomedical applications.

Krzysztof Zakrzewski, M.D., Ph.D., is an associate professor at the Department of Neurosurgery, Polish Mother's Memorial Hospital, Research Institute in Łódź (Poland). His research interests include pediatric neurosurgery with the focus on pediatric neurooncology and neuroradiology.

Emilia Nowosławska is an associate professor at the Department of Neurosurgery, Polish Mother's Memorial Hospital, Research Institute in Łódź (Poland). Her research interests include pediatric neurosurgery with the focus on hydrocephalus and neuroendoscopy.

Received: 17 January 2013

Revised: 28 December 2013 